\title{
Les besoins de circulation et leurs modalités
}

\section{The need for circulation and ways and means}

\author{
J.P. Porcher
}

Délégué régional

Adjoint au Conseil supérieur de la pêche

84, rue de Rennes, 35510 Cesson Sévigné

La première partie de l'article définit la notion de migration et présente les principales espèces migratrices françaises (holobiotiques ou amphibiotiques) dont les cycles biologiques sont illustrés par plusieurs exemples.

La seconde partie détaille les exigences liées à l'aménagement d'obstacles à la migration pour préserver les populations piscicoles migratrices.

La conclusion rappelle la nécessité d'une harmonisation des interventions humaines à l'échelle du bassin hydrographique, afin d'assurer la prise en compte globale des intérêts économiques et écologiques.

The first part of this article defines the idea of migration and introduces the main French migrating (holobiotic and amphibiotic) species whose cycles are illustrated by several examples.

The second part specifies the requirements linked with the disposition of obstacles facing migration for preserving the migratory piscicultural populations.

The conclusion relates to the need for a collective human intervention to the scale of the hydrographic basin so as to ensure that ecological and economic interests are fully taken into account.

\section{Rappel de la notion de migrateur}

Les populations de poissons dépendent très étroitement des caractéristiques de l'habitat du milieu aquatique: celui-ci est, en effet, le support de toutes les fonctions biologiques (reproduction, nutrition, locomotion). Ces fonctions sont, en outre, réglées par le facteur température, d'une importance toute particulière pour des animaux à sang froid.
Trois types de milieu sont indispensables pour le déroulement du cycle de vie d'une espèce :

- une zone de reproduction;

- une zone de production de juvéniles;

- une zone de grossissement pour la production de géniteurs.

Cette remarque a plusieurs conséquences :

1. Si ces trois zones sont contigües, l'espèce sera dite sédentaire. En revanche, si elles sont éloignées les unes des autres dans l'espace, l'espèce est migratrice. 


\title{
Un guide SHF pour la petite hydroélectricité
}

\author{
A small hydro SHF handbook
}

A l'initiative de la Société hydrotechnique de France, une commission spécialisée comprenant à la fois des maitres d'œuvre, des fabricants de machines et des installateurs vient de réaliser un Guide pour la conception, la réalisation, la mise en service et l'exploitation des petits aménagements hydroélectriques. (270 pages).

Cette commission, présidée par M. P. Morand (Compagnie nationale du Rhône), était animée par MM. G. Feillée (Electricité de France) et C. Le Plomb (Compagnie nationale du Rhône).

Destiné aux personnes ou organisations peu familiarisées avec la petite hydroélectricité, le guide, dont le sommaire figure en annexe, comprend deux parties bien distinctes.

La première partie, qui constitue le guide proprement dit, couvre la totalité de l'itinéraire à suivre pour réaliser une installation depuis l'esquisse du projet jusqu'à sa réalisation, sa mise en service et son exploitation. Une série de démarches successives, conduiront le lecteur en fonction de divers critères de choix, soit à une réalisation effective, soit à l'abandon du projet.

Le travail tient compte de la relation essentielle entre les domaines d'application et l'impact économique et social de l'énergie produite dans le contexte local.

Au préalable, afin de bien situer les conditions du placement de l'énergie, sont exposées diverses notions pratiques relatives à l'utilisation de l'énergie ainsi que les contraintes liées à sa qualité.

Puis, sont abordées la méthodologie générale conduisant à une première estimation de la faisabilité d'un projet et la façon de conduire les études (études préliminaires, étude de faisabilité et avant projet détaillé).

S'agissant des matériels, un développement particulier détaille les éléments à prendre en considération pour les garanties à demander aux fabricants ainsi que pour les contrôles permettant de s'assurer de la bonne qualité des matériels fournis, étant entendu que les vérifications de ces garanties et les contrôles imposés ne doivent pas entraîner un coût prohibitif.

Des indications sont ensuite données pour aider à la rédaction de l'appel d'offres (avec un exemple de découpage en lots des équipements électromécaniques) et pour faciliter la comparaison des offres reçues.

Enfin, sont soulignés conseils et mises en garde en vue d'éviter des perturbations dans le déroulement du chantier ainsi que dans la prise en charge de l'installation après le départ des constructeurs.

Un lexique sommaire précise la définition de la terminologie technique utilisée dans le guide lors- qu'elle n'est pas précisée directement dans le texte.

La deuxième partie du document consiste en une série de dix annexes qui développent certaines données évoquées à l'intérieur des chapitres de la première partie. En fonction de ses propres connaissances des problèmes liés à la minihydroélectricité, l'utilisateur du guide aura donc à s'y référer, ou non.

Ces annexes ont :

- soit un caractère purement technique lié à la détermination du projet depuis l'inventaire des ressources hydroélectriques, les besoins d'énergie, le dimensionnement des ouvrages et le choix des machines;

- soit un caractère économique et administratif (étude financière et étude d'impact) permettant d'arriver à la réalisation de l'appel d'offres:

- la dernière annexe donne une liste d'ouvrages récents relatifs à la minihydroèlectricité.

\section{Plan du guide}

Préambule

Ch. I - Présentation du guide - Processus

Ch. II - Présentation des ouvrages

Ch. III - Orientation méthodologique générale pour l'étude d'un projet

Ch. IV - Placement de l'énergie

Ch. V - Conduite des études

Ch. VI - Nature et vérification des garanties

Ch. VIl - Appel d'offres

Ch. VIII - Comparaison des offres

Ch. IX - Exécution et réception des ouvrages

Ch. $\mathrm{X}-$ Exploitation des ouvrages

Terminologie

Annexes

1 - Inventaire des ressources hydroélectriques

2 - Prévisions du développement de la consommation d'énergie électrique

3 - Études sur le terrain

4 - Dimensionnement général des ouvrages

5 - Choix des groupes

6 - Études économique et financière

7 - Étude d'impact

8 - Appels d'offres

9 - Feuilles documentaires pour les petites installations hydrauliques

10 - Bibliographie

En vente à la Société hydrotechnique de France (199. rue de Grenelle, 75007 Paris. Tél. 47.05.13.37) au prix de $250 \mathrm{~F}$. 
Le Président remercie dabord M. FELLLEE de sa présentation des travaux menés par la Commission sur la Minihydroélectricité et des indications qu'il a données sur le rapport fait par M. TRANAIN qui n'a pu participer à la séance.

Il remercie ensuite M. Le PLOMB de son exposé sur les principes et le contenu de l'ouvrage élaboré.

Le Président. - Avant d'ouvrir la discussion, si nous voulions résumer certains aspects essentiels, nous pourrions dire que la volonté du groupe de travail, sous l'impulsion de ses animateurs. a été avant tout d'essayer d'éviter tout d'abord au maître douvrage, quel qu'il soit, de se trouver engagé dans un processus dont il ne sache plus sortir si, à un moment donné, il apparaissait que l'ouvrage projeté aurait, pour des raisons soit techniques, soit économiques, été difficilement réalisable, voire même irrẻalisable. Par conséquent, il était important de marquer des étapes, voire même des possibilités de retour en arrière d'un certain processus qui les traduise, de manière à pouvoir s'arrêter ou «corriger le tir " si. à un certain moment. le résultat d'études relativement légères et, dans la progression de la préparation du projet, d'études de plus en plus lourdes montrait que l'on est engagé sur une mauvaise voie.

D'autre part, nous avons cherché aussi à éviter. et ceci au bénéfice réciproque du maitre d'ouvrage et des constructeurs, que des maîtres d'ouvrage mal informés n'en demandent trop. ce qui a évidemment pour premier effet daccroitre les coûts, mais qui peut aussi introduire des distorsions de concurrence, ceci afin qu'on n'aille pas demander parfois des choses farfelues, peut-être même simplement en matière d'essais, à des constructeurs. Jai le souvenir d’avoir dvu 9 énormes pompes, selon la volonté du maittre d'ouvrage et de son ingénieur, dans un pays africain, passer en rang d'oignon sur le banc d'essai du CETIM de Nantes, qui était le seul à pouvoir les accueillir en France. Toutes ces pompes sont allées de Chartres à Nantes de Nantes à Chartres, puis de Chartres à Rouen pour le simple plaisir de les avoir essayées une par une, alors qu'elles étaient rigoureusement identiques et relevaient de modèles parfaitement classiques du constructeur. C'est aussi ce genre de chose que nous avons voulu. sinon éviter, du moins atténuer en faisant en sorte qu'un maître d'ouvrage aussi bien informé que possible n'aille pas demander des choses que nous pourrions qualifier de superfétatoires.

Nous allons maintenant aborder la discussion. Qui demande la parole?

M. GIRAULT. - Est-ce que ce Guide va donner lieu à l'établissement d'un cahier des charges simplifié ?

M. Le Président. - Je me tourne vers les responsables du groupe de travail, mais aussi vers le Président BANAL.

L'idée initiale était de faire un document: on avait parlé de normalisation au début, puis nous nous sommes aperçu en fait que vouloir normaliser les petites centrales hydroélectriques était peut-être beaucoup trop ambitieux et en même temps que ce n'était peut-être pas faisable.

De la même façon, en ce qui concerne un cahier des charges simplifié, je m'interroge un peu, parce que, dans un cahier des charges, il y a un certain nombre de principes, qui ressortent d’ailleurs assez clairement de la deuxième partie du Guide et surtout de ses annexes: mais de là à vouloir présenter un modèle. alors que nous nous adressons á des maîtres d'ouvrage extrêmement hétérogènes, puisque nous nous adressons à des maîtres d'ouvrage de tous les pays du monde. qui ont des traditions techniques très variées: les traditions techniques françaises. anglo-saxonnes, allemandes, etc. en matière de spécifications ne sont pas les mêmes.

Nous avons cherché à définir des principes qui aident à ce que les choses soient comparables. De lá à déboucher sur un cahier des charges simplifié, je ne sais pas ce qu'en pense le Président BANAL, mais je craindrais que ce ne soit peut-être un peu à côté de notre objectif.
M. BANAL. - Je répondrai volontiers que si vraiment un besoin se manifeste, la Société Hydrotechnique de France neéludera pas ses responsabilités ni son désir d'ètre utile.

Je pense qu'un des intérêts de linformation qui est lancée aujourd hui par ce Guide est justement de provoquer les réactions des lecteurs. Les auteurs ont dit qu'il contenait des erreurs. des coquilles, etc. Que l'on nous dise les corrections a faire. nous en seront trés contents. Mais je pense que l'on peut aller plus loin, et dire que s'il y a des compléments à apporter, de nouvelles directions dans lesquelles on puisse aller. pourquoi pas? La SHF écoutera à partir d’aujourd'hui les réactions. les commentaires qui vont être donnés sur ce Guide.

Puisque jai la parole, je dirai que jai une petite critique à faire: dans le Guide. il n'est pas question des mini-usines marémotrices: c'est un sujet qui n’a pas été abordé et qu'on aurait pu traiter. Je me demande s'il ne serait pas intéressant de faire un complément à l'usage des personnes des régions littorales qui voudraient faire des mini-usines maremotrices.

M. Le Président. - Je crois qu'il y a là un champ d'action fort intéressant, mais qui risque de soulever parfois localement des polémiques.

M. GAUTHERON. - Une petite usine marémotrice avait été envisagée à St Pierre et Miquelon, mais la marée étant très faible, de l'ordre du métre. l'opération n'était pas économiquement envisageable.

M. MONITION, - Le projet en tant quopération de petite marémotrice, opération encouragée par la Commission des communautés Européennes / D G 17, n'est pas abandonné. II pourrait être transféré en Bretagne où les marées sont de plus grande amplitude. Par contre, des aérogénérateurs pourraient ẻtre proposés pour St Pierre et Miquelon doté de ressources éoliennes importantes.

M. FEILLEE. - Le guide ne traite pas des petits groupes expérimentaux (vagues, courants marins, etc.).

M. Le Président. - De toute façon. ce Guide se voulant essentiellement un instrument d'utilisation pratique, il traite d'aspects qui sont du domaine en cours. Les projets qui en sont encore au stade de la recherche, voire du développement technologique n'avaient pas leur place, compte tenu de l'esprit dans lequel ce document a été préparé.

M. GIRAULT. - Electrobras est en train de développer un guide du même type au Brésil. N'y a-t-il pas une çoopération possible à envisager?

M. Le Président. - Bien que ne connaissant pas le projet, la S.H.F. accepterait d'y participer et demande à chacun de participer au maximum à la diffusion internationale du guide.

M. ARGEnSON. - Le guide ne fait-il pas double emploi avec ceux publiés aux U.S.A. par des Universités?

\section{Le Président. - La bibliographie a èté faite.}

M. FEILLEEE. - Tous les guides n'ont pas le même but. Par exemple, celui de l'UNIDO est plutôt orienté vers la recherche de financement. Les guides américains sont destinés à des centrales plus importantes jusquà 20 ou $30 \mathrm{MW}$ et donnent plus des coûts que des méthodes à suivre. De même. le projet de la CEI se limite aux matériels. Ces divers ouvrages sont des compléments et ne font donc pas double emploi avec notre guide.

M. Le Président. - Je crois que, de toute façon. ce qu'il est important de noter, c'est que nous avons voulu aborder le problème en considérant l'opération d'aménagement dans son ensemble; c'est peut-être ce qui fait le plus l'originalité du guide qui vous est présenté aujourd'hui. 
- Ceci suppose d'abord une bonne connaissance des espèces présentes, connaissance qui n'est pas acquise à l'heure actuelle pour la totalité du réseau hydrographique.

Ainsi, tout récemment, des observations réalisées sur des stations de contrôle des migrations ou par des inventaires piscicoles ont-elles permis en Bretagne et Basse-Normandie de repérer des populations naturelles d'espèces migratrices dans des bassins fluviaux où elles n'étaient pas soupçonnées.

Une enquête précise, et des investigations préalables devront dans la quasi totalité des cas précéder la conception de l'ouvrage de franchissement.

- Le dispositif devra permettre le passage des différentes espèces et être adapté à leur comportement ou leurs performances respectives (capacité ou non de saut, vitesses de nage, zones de rassemblement en aval de l'obstacle, etc.). Ceci pourra conduire à équiper un obstacle de plusieurs passes à poissons pour prendre en compte des espèces différentes, ou s'adapter à des conditions hydrauliques variables suivant les saisons.

- Le calendrier de fonctionnement de l'ouvrage devra assurer son efficacité pendant toute la durée de la migration, aussi bien à la montée qu'à la descente. Il apparaît que sur de nombreux bassins, en raison de la multiplicité des espèces présentes, les migrations peuvent couvrir la quasi-totalité de l'année, avec une seule période creuse en plein étiage, très limitée dans le temps certaines années en fonction des conditions hydrologiques.

\subsubsection{L'ouvrage de franchissement et son environne- ment doivent respecter l'intégrité physique des migrateurs}

- A la montée, les migrateurs ne doivent pas subir de dommage physique lors du franchissement de l'obstacle. Pour cette raison, on peut être amené à équiper de dispositifs de franchissement des ouvrages de hauteur modérée qui ne constituent un obstacle que pendant une courte période de l'année : ainsi, en Bretagne, des petits barrages de moulins, aisément franchissables en eaux moyennes ou en crue bloquent les migrations de castillons (saumons de remontée estivale et automnale), les années d'étiage tardif.

Les tentatives de franchissement par saut sont à l'origine de blessures des géniteurs. Cette situation est aggravée :

- directement par concentration des individus à l'aval de l'ouvrage, entrainant un terrain pathologique favorable sur des poissons déjà fragilisés physiologiquement par le processus de maturation sexuelle;

- indirectement par l'augmentation récente du nombre de salmonicultures industrielles qui peuvent être à l'origine d'une dissémination de germes pathogènes.

- A la descente, le dispositif de franchissement doit détourner le poisson des installations susceptibles de le blesser (grilles de prise d'eau, turbines, etc.). Certaines espèces apparaissent très vulnérables: exemple de l'anguille sur laquelle on peut observer des mortalités importantes au passage des turbines (grande taille, dévalaison au voisinage du fond).
Les ouvrages devront prendre en compte non seulement les juvéniles, mais également les reproducteurs après la fraie pour les espèces à reproductions multiples.

\subsubsection{Le dispositif de franchissement doit respecter les exigences biologiques de l'espèce}

- Il ne doit pas entraîner de retard excessif à la migration: pour les espèces migratrices potamotoques, un retard dans la montée des géniteurs peut se traduire par une impossibilité de gagner à temps les zones de reproduction, ou par une mauvaise répartition des géniteurs sur ces zones, entraînant des concentrations locales trop importantes de frayères, et par conséquent des mortalités élevées au stade juvénile.

A la descente des juvéniles, un retard peut perturber le comportement migratoire (accès aux zones de grossissement marines).

- La gestion des barrages doit éviter d'ajouter à l'obstacle physique qu'ils constituent un obstacle de nature biologique (qualité, température des eaux restituées, répartition spatiale des salinités pour les barrages de fond d'estuaire...).

\subsubsection{La libre circulation n'est jamais définitivement acquise}

Tout changement de conditions d'environnement (hydrologie, modelé du lit du cours d'eau,...) ou des conditions d'exploitation de l'ouvrage (niveau de prise d'eau, de restitution, modification des installations,...) peut rendre un obstacle infranchissable, ainsi :

- les moulins d'autrefois, équipés de roues, utilisaient souvent une faible partie du débit du cours d'eau à certaines périodes de l'année. En outre, les règlements prescrivaient un chômage hebdomadaire, et la puissance motrice n'était souvent utilisée que dans la journée... De ce fait, le déversoir de prise d'eau était alimenté à intervalles réguliers et franchissable par les migrateurs. Le remplacement des roues par des turbines, utilisant souvent un débit plus élevé et fonctionnant automatiquement 24 heures sur 24 peut assécher les déversoirs pendant plusieurs mois consécutifs et rendre infranchissable un obstacle qui ne présentait pas de difficulté à l'origine.

- sur les cours d'eau navigables, le remplacement des portes d'écluses en bois comportant de nombreuses fuites, par des portes métalliques étanches peut interrompre totalement la migration des civelles.

Chaque obstacle pouvant, à lui seul, condamner l'existence d'une population, il convient donc d'exercer une vigilance constante vis-à-vis de la libre circulation des migrateurs.

\subsubsection{Les projets d'aménagement doivent être pensés en fonction de l'objectif "libre circulation"}

Dans certains cas, l'aménagement pourra être conçu de telle sorte qu'il ne constitue pas un obstacle : un seuil de hauteur importante peut être remplacé par 2 ou 3 seuils de hauteur modérée, ou être équipé dès sa réalisation d'un chenal latéral pour les migrations, etc. 


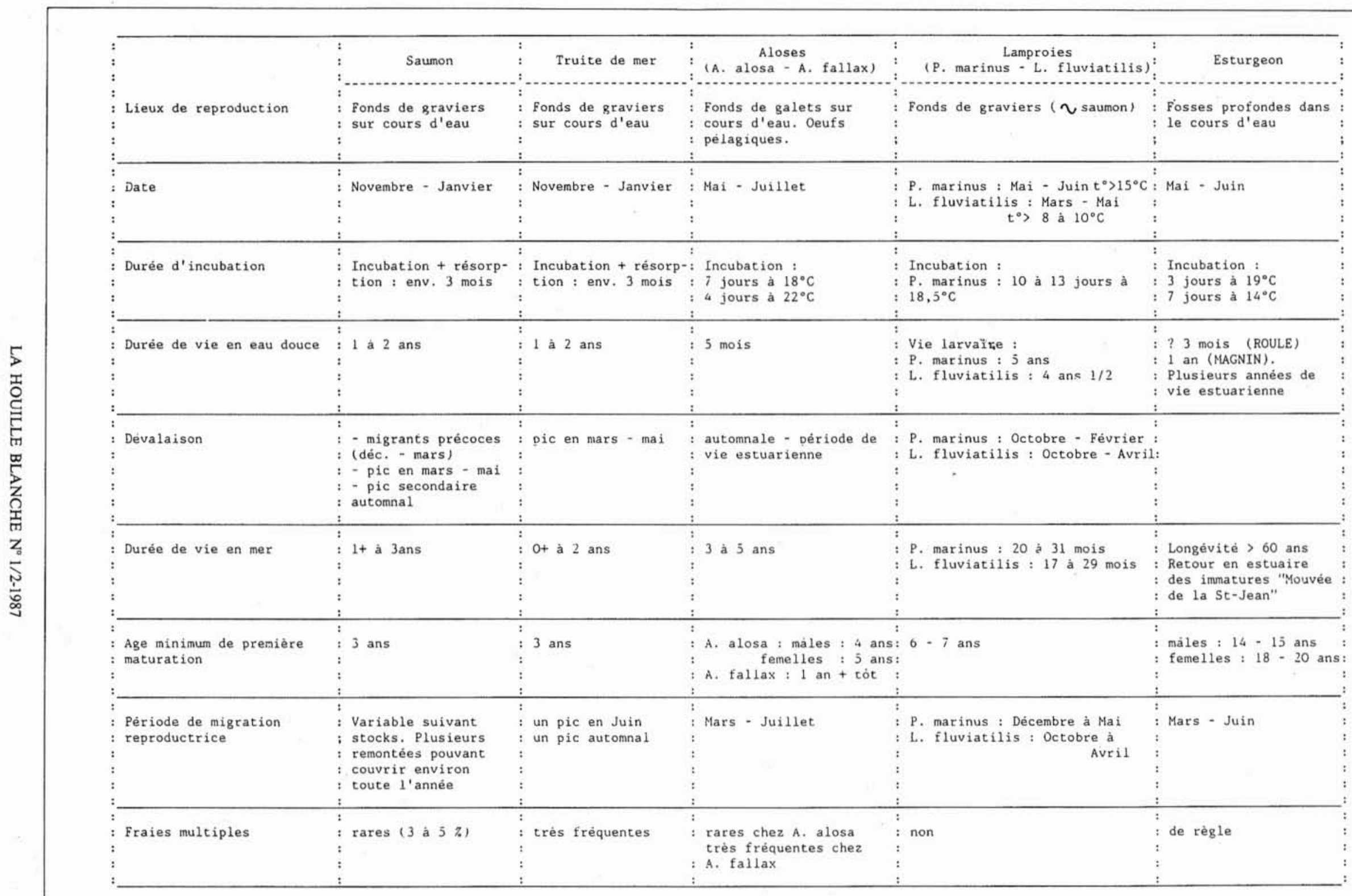

Figure 1. - Principales caractéristiques des cycles biologiques des migrateurs potamotoques 


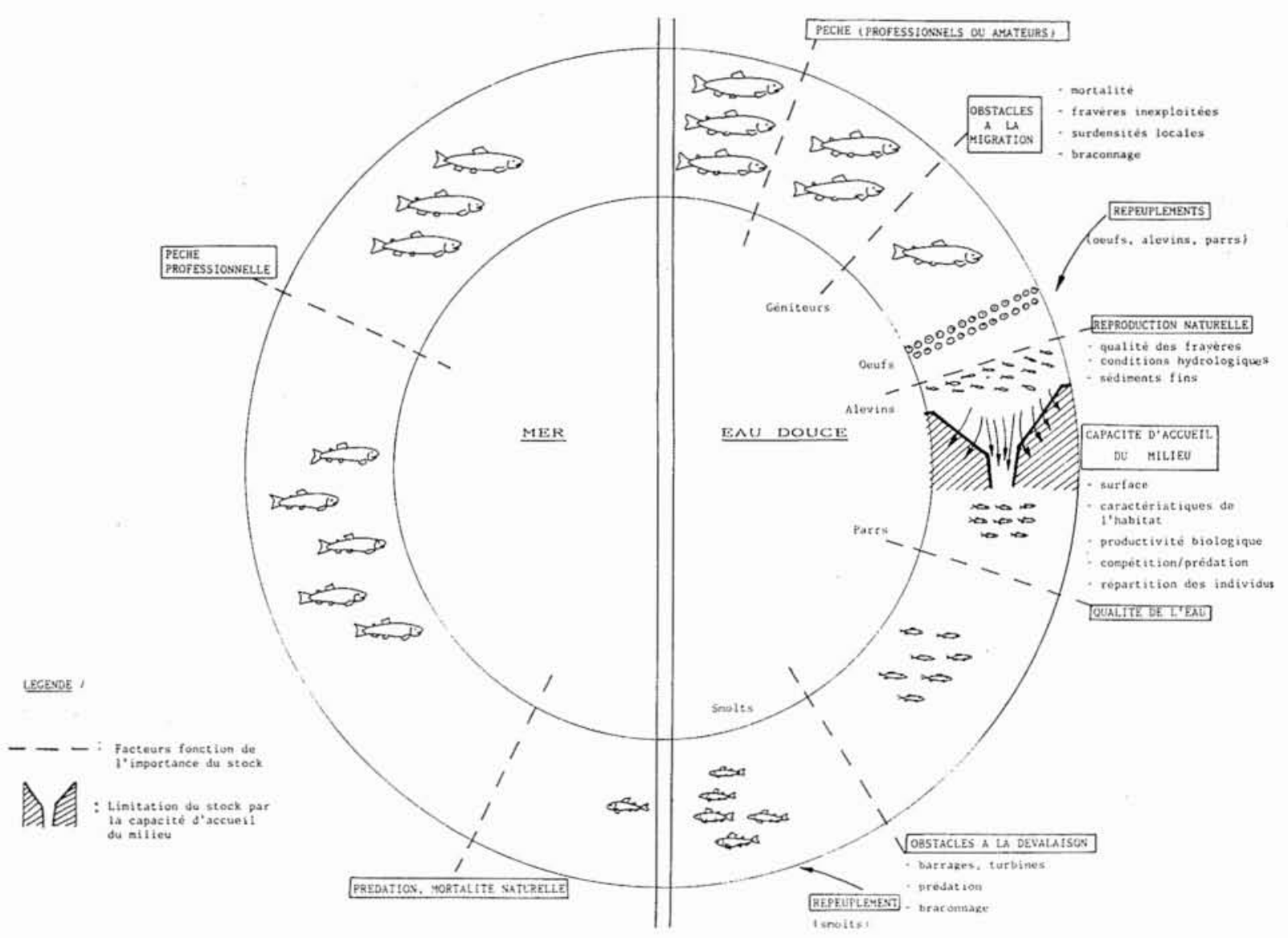

Figure 2. - Facteurs de régulation des stocks de saumon atlantique

\section{Interactions entre activités humaines et popu- lations piscicoles migratrices : pour une ges- tion globale et cohérente au niveau du bassin}

L'étude des cycles biologiques des espèces migratrices montre que si la libre circulation constitue une condition nécessaire au maintien des populations, de nombreux autres facteurs interviennent dans la régulation des effectifs. L'examen des facteurs intervenant sur les populations de saumon (fig. 2) indique que l'abondance du stock est conditionnée par des caractéristiques du milieu naturel et par des interventions humaines.

La plupart de ces facteurs opèrent une réduction grossièrement proportionnelle (en première approximation) aux effectifs présents, avec toutefois des pourcentages de réduction pouvant atteindre $100 \%$ (cas d'un obstacle infranchissable, d'une dégradation excessive de la qualité de l'eau).

Les facteurs trophiques ou climatiques qui interviennent au niveau de la phase marine jouent un rôle important dans la régulation des stocks. Toutefois, il ne faut pas négliger la place essentielle tenue par la capacité de production de juvéniles du milieu eau douce, qui est à la base de ce stock. Les paramètres mis en jeu dans cette phase restent les plus accessibles pour la mise en œuvre d'une politique de gestion des populations migratrices, qui peut se caractériser par :

- le maintien du cycle biologique des espèces migratrices;

- la recherche du stock optimum (en particulier au niveau du potentiel de reproduction) dans le réseau hydrographique concerné.

Une gestion cohérente d'un peuplement des migrateurs devra donc s'attacher :

1) A diminuer autant que possible des facteurs de réduction du stock en limitant les nuisances ou (si nécessaire) les prélèvements exercés sur ce stock pour conserver un effectif optimum de géniteurs

2) A augmenter les capacités d'accueil et de production du milieu :

- par une mise à disposition de nouvelles zones de production (suppression d'obstacles à la migration);

- par une sauvegarde, une restauration ou un aménagement de zones existantes (entretien de cours d'eau, lutte contre la pollution, récupération de frayères ennoyées par des retenues...).

Un tel programme ne peut être conduit qu'à l'échelle du bassin tout entier puisque les facteurs à prendre en compte à quelque niveau du bassin hydrographique que 
ce soit ont des répercussions sur le stock global du réseau hydrographique.

Le maintien ou la restauration des habitats indispensables au bon déroulement du cycle biologique d'une espèce migratrice sont des objectifs qui s'insèrent étroitement dans le contexte des activités humaines et présentent des implications économiques immédiates :

- activités industrielles, rejets domestiques/qualité de l'eau;

- aménagements hydrauliques, hydro-agricoles/conservation des biotopes;
- ennoyage par des retenues de barrages de zones de reproduction et de production de juvéniles;

- extraction de granulats dans des zones indispensables pour la reproduction, instabilité subséquente du lit du cours d'eau;

- etc.

La cohérence d'une gestion des populations de migrateurs passe donc par la prise en compte des intérêts écologiques et économiques du bassin hydrographique pris dans son ensemble. sant.

M. Le Président remercie l'ảuteur pour son exposé très intéres-

M. LEGRAND demande si les différences de niveau parfois relativement importantes (50 à $80 \mathrm{~cm}$ ) et rapides dues aux barrages situés en amont de la Loire peuvent apporter des perturbations sur la migration des poissons.

Dans sa réponse $M$. PORCHER explique que les variations de niveau ne sont pas uniquement causées par les barrages mais peuvent être dues à des phénomènes naturels tels que les crues. On sait de façon précise que les migrations sont fonction du débit, qui est un facteur déclenchant. Un étiage prolongé dû à un débit réservé maintenu tardivement en automne peut bloquer la migration. De même la présence d'éclusées peut inciter la remontée à un moment peu favorable au point de vue température par exemple. Ceci tend vers l'élaboration de plan d'aménagement destiné à respecter les intérêts de chaque partie présente au fil de l'eau tout en évitant de perturber les problèmes de migration.

$M$. Le Président fait remarquer que c'est un élément important et la lâchure d'eaux à un moment inopportun peut entraîner des perturbations dans les cycles de croissance piscicole.

M. PORCHER signale que s'ajoutent à celà des problèmes de qualité d'eau et de température différentes

M. MASSON s'interroge sur la contradiction apparente entre les possibilités migratrices importantes des jeunes anguilles (franchissement d'obstacles verticaux, migration en dehors du lit des cours d'eau) et le dépeuplement effectif des rivières éloignées de la mer. Ce dépeuplement nécessite des alevinages à partir de civelles provenant du Sud-Ouest.

M. PORCHER explique que l'observation de la distribution de l'anguille dans les bassins bretons montre une corrélation très nette entre la densité de la population présente et l'éloignement de la mer.

Deux phénomènes entrent en jeu :

- un phénomène naturel : il est tout à fait normal que les populations de civelles soient plus importantes près de la mer; - un phénomène dû aux obstacles à la migration qui limitent la colonisation des bassins amont. Par exemple les anciennes écluses de navigation permettaient le passage des civelles par l'existence de fuites au niveau des portes en bois. Sur un certain nombre de cours d'eau, ces portes ont été remplacées par des portes métalliques étanches qui empêchent le passage des civelles.

Cet exemple de modification qui semble mineure peut concerner toute une population. 